\title{
The efficacy of selective nerve root block for the long-term outcome of postherpetic neuralgia
}

\author{
A Ram Doo ${ }^{1,2}$, Jin-Wook Choi ${ }^{1}$, Ju-Hyung Lee ${ }^{3}$, Ye Sull Kim', Min-Jong Ki ${ }^{1}$, Young Jin Han ${ }^{1,2}$, and Ji-Seon Son ${ }^{1,2}$ \\ ${ }^{1}$ Department of Anesthesiology and Pain Medicine, Chonbuk National University Medical School, Jeonju, Korea \\ ${ }^{2}$ Research Institute of Clinical Medicine of Chonbuk National University-Biomedical Research Institute of Chonbuk National \\ University Hospital, Jeonju, Korea \\ ${ }^{3}$ Department of Preventive Medicine, Chonbuk National University Medical School, Jeonju, Korea
}

Background: Several nerve blocks can reduce the incidence of postherpetic neuralgia (PHN) as well as relieve acute zoster-related pain, but the long-term outcome of PHN has not been clearly determined. This study investigated the efficacy of selective nerve root block (SNRB) for herpes zoster (HZ) on the long-term outcome of PHN

Methods: We prospectively conducted an interview of patients who had undergone an SNRB for HZ from January 2006 to December 2016 to evaluate their long-term PHN status. The relationship between the time from $\mathrm{HZ}$ onset to the first SNRB and the long-term outcome of PHN was investigated.

Results: The data of 67 patients were collected. The patients were allocated to acute (SNRB $\leq 14$ days, $n$ $=16)$ or subacute $(\mathrm{SNRB}>14$ days, $\mathrm{n}=51)$ groups. The proportions of cured patients were $62.5 \%$ and $25.5 \%$ in the acute and subacute groups $(P=0.007)$, respectively. In logistic regression, an SNRB $>14$ days was the significant predictor of PHN (adjusted odd ratio, 3.89; 95\% confidence interval, 1.02-14.93; $P=0.047$ ). Kaplan-Meier analysis revealed that time from the SNRB to the cure of PHN was significantly shorter in the acute group $(2.4 \pm 0.7 \mathrm{yr})$ than in the subacute group $(5.0 \pm 0.4 \mathrm{yr} ; P=0.003)$.

Conclusions: An early SNRB during the acute stage of HZ (within 14 days) appears to decrease the incidence and shorten the duration of PHN, with a median of 5.0 years of follow-up. (Korean J Pain 2019; 32: 215-22)

Key Words: Follow-Up Studies; Ganglia, Spinal; Herpes Zoster; Incidence; Logistic Models; Nerve Block; Neuralgia, Postherpetic; Pain.

\section{INTRODUCTION}

Herpes zoster (HZ) is a varicella-zoster virus-induced dis- ease, which results from the reactivation of the dormant virus, which resides latently in the spinal dorsal root ganglion (DRG) or cranial sensory ganglion after primary vari-

Received April 17, 2019. Revised June 4, 2019. Accepted June 10, 2019.

Correspondence to: Ji-Seon Son

Department of Anesthesiology and Pain Medicine, Chonbuk National University Medical School, 20 Geonji-ro, Deokjin-gu, Jeonju 54907, Korea Tel: +82-63-250-1241, Fax: +82-63-250-1240, E-mail: sjs6803@jbnu.ac.kr

ORCID: https://orcid.org/0000-0002-6672-4576

(a) This is an open-access article distributed under the terms of the Creative Commons Attribution Non-Commercial License (http:// creativecommons.org/licenses/by-nc/4.0/), which permits unrestricted non-commercial use, distribution, and reproduction in any medium, provided the original work is properly cited.

(C) The Korean Pain Society, 2019 
cella infection earlier in life. HZ results in a painful vesicular rash in a dermatomal distribution corresponding to the DRG involved. Postherpetic neuralgia (PHN) is the most common and troublesome complication of $\mathrm{HZ}$ and presents as painful symptoms that persist for more than 90 days after rash onset [1]. The estimated incidence of PHN has been reported as $12.5 \%-38.4 \%$ in the literature, and this incidence increases with age [1-3]. PHN typically manifests chronic intractable neuropathic pain that persists for months or even years; it can result in decreased quality of life and increased incidence of mood or sleep disorders [2].

Even though the pathophysiology of PHN is poorly understood, it is well-known that severe, uncontrolled zoster-related pain (ZRP) during the acute stage of $\mathrm{HZ}$ is one of the major risk factors for development of PHN. In cases of ZRP which is intractable and severe, in spite of various pharmacologic treatment including antiviral agents, corticosteroid and analgesics, such as nonsteroidal anti-inflammatory drugs and opioids, interventional treatment can be helpful for relieving symptoms. Several studies have demonstrated that the application of a nerve block such as a somatic nerve block, paravertebral block, transforaminal epidural block, continuous epidural block through a catheter, or a sympathetic nerve block during the acute stage of HZ shortens the duration of ZRP, decreases the intensity of acute pain, and reduces the incidence of PHN [4-6]. However, the long-term clinical outcomes of PHN, with or without interventional treatment, have not been clearly determined.

The DRG is the original site of central and peripheral nervous system sensitization phenomenon associated with the occurrence of PHN as well as development of HZ induced by reactivation of the dormant virus. Therefore, the DRG is a primary target for intervention to manage ZRP. A selective nerve root block (SNRB) may be the theoretically most effective method of drug delivery to the DRG with a small volume of injectate compared to other nerve blocks.

We conducted a combined retrospective and prospective study to investigate the long-term efficacy of an SNRB for PHN in patients with HZ. We hypothesized that application of an early SNRB during the acute stage of $\mathrm{HZ}$ would decrease the incidence of persistent PHN and the severity of persistent pain during long-term follow-up.

\section{MATERIALS AND METHODS}

This combined retrospective and prospective study was approved by the Institutional Review Board of the Chonbuk National University Hospital (No. CUH 2019-04-049), which waived the requirement for informed consent. The study was conducted in accordance with Good Clinical Practice and the International Conference on Harmonization guidelines, and in conformity with the ethical principles of the Declaration of Helsinki. Medical records of $456 \mathrm{pa}-$ tients who visited the pain clinic for ZRP, at a tertiary care university hospital during the period from January 2006 to December 2016, were retrospectively reviewed. Among them, 186 patients who had undergone the SNRB procedure for treating ZRP, involving a single dermatomal area, were identified. Patients' demographic data, zoster-involved area, current medications, details of the SNRB procedure and the duration to the first SNRB procedure from zoster onset were recorded. Patients who received other procedures, such as a sympathetic block, intercostal block, paravertebral block, or radiofrequency therapy, in addition to an SNRB, were excluded. Patients who received an SNRB for PHN greater than 90 days after the onset of ZRP were also excluded. The subject flow diagram is presented in Fig. 1.

We prospectively conducted a personal interview with 102 patients via telephone call to evaluate their long-term PHN-related status between September 2017 and October 2017 (by a single investigator, Dr. JW Choi). The patients were asked to classify their current severity of PHN using a 4-step severity grading system in which patients chose between "relieved", "improved", "sustained", or "aggravated". The details of the grading system are as follow: "relieved", no pain; "improved", pain is reduced but remains; "sustained", painful with no change in pain after treatment; and "aggravated", more painful after treatment. The patients who denied any PHN-related persistent pain which was expressed as "relieved" were categorized as the cured group, and the patients with persistent PHN which means "improved", "sustained" or "aggravated" were categorized as the non-cured group.

\section{SNRB procedures}

The SNRB procedures were conducted with guidance with a fluoroscope (ARCADIS Orbic ${ }^{\circledR}$; Siemens AG, Erlangen, 


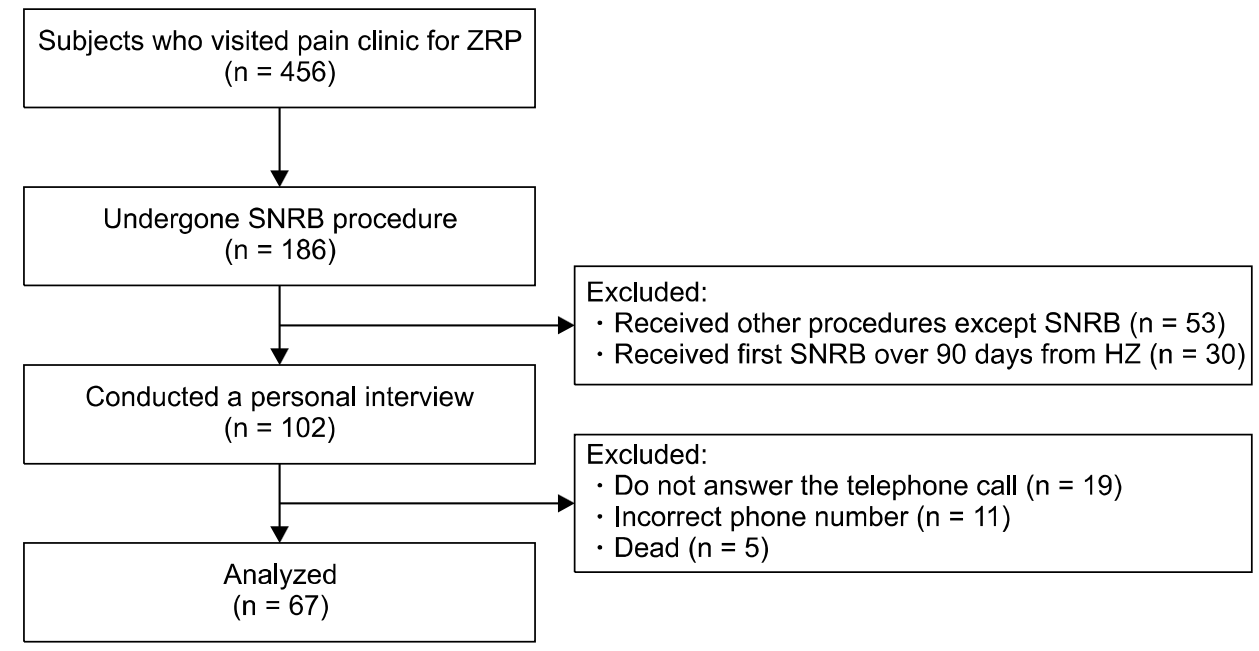

Fig. 1. Subjects flow diagram. ZRP: zoster-related pain, SNRB: selective nerve root block, $\mathrm{HZ}$ : herpes zoster.
Germany) as described previously [7]. For thoracic procedures, the patients were placed in a prone position. After the usual sterile preparation, once the level was identified, the fluoroscope was rotated ipsilateral obliquely to bring the spinous process and head of the ribs medially, and the needle was introduced just inferolateral to the pedicle at the level of the pathology using tunnel vision technique under fluoroscopic guidance. When the needle approached the intervertebral foramen, the final position of needle tip was adjusted and confirmed by an anteroposterior (AP) and lateral fluoroscopic view. The needle tip was seen to lie just on the lateral laminar border on the AP view, and the lower posterior portion of the intervertebral foramen on the lateral view. A total of 0.5 to $1.5 \mathrm{~mL}$ of contrast dye (Omnipaque; GE Healthcare, County Cork, Ireland) was injected to confirm appropriate dye spreading around the DRG. After confirming negative vascular and epidural uptake, $1.5 \mathrm{~mL}$ of $0.5 \%$ ropivacaine containing $10 \mathrm{mg}$ triamcinolone was administrated.

For the lumbosacral procedure, the fluoroscopy was rotated ipsilateral side obliquely in order toobtain a clear image of the space between the pedicle and the nerve root to be treated. The needle was advanced just inferior to the pedicle adjacent to the nerve root. The final position of needle was seen just inferior to the pedicle on the AP view and just anterior to the zygapophyseal joint on the lateral image. After confirming negative vascular and epidural uptake, $2 \mathrm{~mL}$ of $0.2 \%$ ropivacaine containing $10 \mathrm{mg}$ triamcinolone was administrated. For the cervical procedure, the anterolateral approach in a supine position was used. After oblique angulation of the fluoroscopic ray, a needle with a bent tip was advanced toward the medial side of the superior articular process just posterior to the foramen. When the needle contacted the superior articular process, the needle was carefully advanced ventromedially toward the posterior portion of the foramen. After confirming negative vascular and epidural uptake using contrast dye, $1 \mathrm{~mL}$ of $0.2 \%$ ropivacaine and $10 \mathrm{mg}$ triamcinolone was administered. The dose and concentration of local anesthetics were adjusted for a profound analgesia with minimal motor block in our standard practice.

All procedures were performed by a single skilled pain physician (Dr. JS Son) with with more than 15 years of experience in interventional pain management.

\section{Statistics}

All descriptive statistics are expressed as mean \pm standard deviation, median (interquartile range, IQR), percentage, or the number of patients. First, we compared the patient demographics between the cured and non-cured groups. Continuous variables were analyzed with the Student $t$-test or Mann-Whitney rank-sum test after a normality test. And categorical variables were analyzed using Fisher exact test.

Second, we investigated the relationship between the time elapsed from $\mathrm{HZ}$ onset to the first SNRB procedure and the probability of persistent PHN to prove our hypothesis that the application of an early SNRB during the acute stage of $\mathrm{HZ}$ would decrease the incidence of persistent PHN and the severity of persistent pain. The time elapsed from $\mathrm{HZ}$ onset to first SNRB procedure associated with the 
probability of persistent PHN was integrated into a logistic regression model, and a receiver operating characteristic (ROC) curve was used to estimate the cut-off value.

Third, we rearranged the allocated patients according to the cut-off value, and compared the two groups. The logistic regression analysis was performed to determine the degree of the variables' association with PHN, described using the odds ratio (OR) and 95\% confidence interval (CI). Finally, the distribution of each time-to-event efficacy end point was accessed by the Kaplan-Meier survival method, and differences between the two groups were compared using the log-rank test. All statistical analyses were performed using SigmaPlot ver. 12.5 (Systat Software Inc., San Jose, CA), and $P$ values $<0.05$ were considered statistically significant.

\section{RESULTS}

Among 102 patients enrolled, we analyzed 67 patients who had completely responded to the interview in this study, 23 from the cured group and 44 from the non-cured group (No. of patients who had reported as "relieved" = 23, "improved" = 36, "sustained" = 7, and "aggravated" = 1), with a median of 5.0 years of surveillance for HZ (ranging from 1 to 8 years). Patient characteristics are described in Table 1. The median time elapsed from $\mathrm{HZ}$ onset to the first SNRB procedure was 20.0 days (IQR, 12.0-36.0 days) in the cured group, which was significantly shorter than that in the non-cured group (median, 30.0; IQR, 19.0-49.8; $P=0.032$ ) (Table 1).

We created an ROC curve (area under the curve [AUC],

Table 1. Patient Demographics

\begin{tabular}{|c|c|c|c|}
\hline Variable & Cured group $(n=23)$ & Non-cured group $(n=44)$ & $P$ value \\
\hline No. of patient & Relieved $=23$ & $\begin{aligned} \text { Improved } & =36 \\
\text { Sustained } & =7 \\
\text { Aggravated } & =1\end{aligned}$ & \\
\hline Age (yr) & $63.0(57.0-72.0)$ & $67.0(61.5-71.8)$ & 0.552 \\
\hline Sex (male/female) & $7 / 16$ & $23 / 21$ & 0.088 \\
\hline Involved dermatome & & & 0.689 \\
\hline Cervical & $2(8.7)$ & $2(4.6)$ & \\
\hline Thoracic & $19(82.6)$ & $36(81.8)$ & \\
\hline Lumbosacral & $2(8.7)$ & $6(13.6)$ & \\
\hline Direction (right/left) & $10 / 13$ & $22 / 22$ & 0.803 \\
\hline \multicolumn{4}{|l|}{ NRS score at first visit $(0-10)$} \\
\hline At rest & $6.0 \pm 2.2$ & $6.6 \pm 2.0$ & 0.355 \\
\hline During pain attack & $6.5 \pm 2.1$ & $6.9 \pm 2.1$ & 0.535 \\
\hline \multicolumn{4}{|l|}{ Medications } \\
\hline Tricyclic antidepressants & $2(8.7)$ & $9(20.5)$ & 0.307 \\
\hline Anticonvulsant & $16(69.6)$ & $31(70.5)$ & 0.837 \\
\hline Analgesics including opioids & $15(65.2)$ & $26(59.1)$ & 0.822 \\
\hline \multicolumn{4}{|l|}{ Underlying disease } \\
\hline Hypertension & $6(26.1)$ & $12(27.3)$ & 0.917 \\
\hline Diabetes mellitus & $1(4.4)$ & $4(9.1)$ & 0.483 \\
\hline Respiratory disease & $3(13.0)$ & $3(6.8)$ & 0.397 \\
\hline Stroke & $1(4.4)$ & $1(2.3)$ & 0.636 \\
\hline Chronic kidney disease & $1(4.4)$ & $0(0)$ & $\mathrm{N} / \mathrm{A}$ \\
\hline Time elapsed from $\mathrm{HZ}$ onset to first SNRB (day) & $20.0(12.0-36.0)$ & $30.0(19.0-49.8)$ & $0.032^{*}$ \\
\hline No. of repeated SNRB procedure & $3.0(2.0-4.0)$ & $4.0(2.0-6.0)$ & 0.231 \\
\hline Time elapsed from $\mathrm{HZ}$ onset to interview (yr) & $5.0(3.0-5.0)$ & $4.0(2.0-5.0)$ & 0.200 \\
\hline
\end{tabular}

Values are presented as number only, median (range), number (\%), or mean \pm standard deviation. Cured group included the patients who stated themselves to be "relieved" (no pain), and non-cured group included the patients with persistent postherpetic neuralgia described as "improved", "sustained", or "aggravated" using 4-step severity grading system.

NRS: numeric rating scale, HZ: herpes zoster, SNRB: selective nerve root block, N/A: not available.

${ }^{*} P<0.05$ by Mann-Whitney rank-sum test between cured and non-cured groups. 
0.658; 95\% CI, 0.514-0.802; $P=0.035)$ to establish the cut-off value of the time elapsed from $\mathrm{HZ}$ onset to the first SNRB predictive of persistent (non-cured) PHN (Fig. 2). The cut-off value was 14.5 days (sensitivity $=86.4 \%$ and specificity $=43.5 \%)$. The patients were reallocated to one of two groups, which were the acute (SNRB performed within 14 days, $\mathrm{n}=16$ ) and subacute (SNRB performed at more than 14 days, $n=51$ ) groups, according to the calculated cut-off value. The mean time (in days) elapsed from $\mathrm{HZ}$ onset to the first SNRB were $11.3 \pm 3.1$ and $39.8 \pm$ 19.2 in the acute and subacute group, respectively $(P<$ 0.001 by the Student $t$-test). Patient-reported long-term severity of PHN was significantly different in the two groups $(P=0.012)$ (Fig. 3). The proportions of cured (relieved) patients were $62.5 \%$ and $25.5 \%$ in the acute and subacute groups, respectively $(P=0.007)$.

In logistic regression analysis, variables with statistical significance in univariate analysis were supposed to be as-

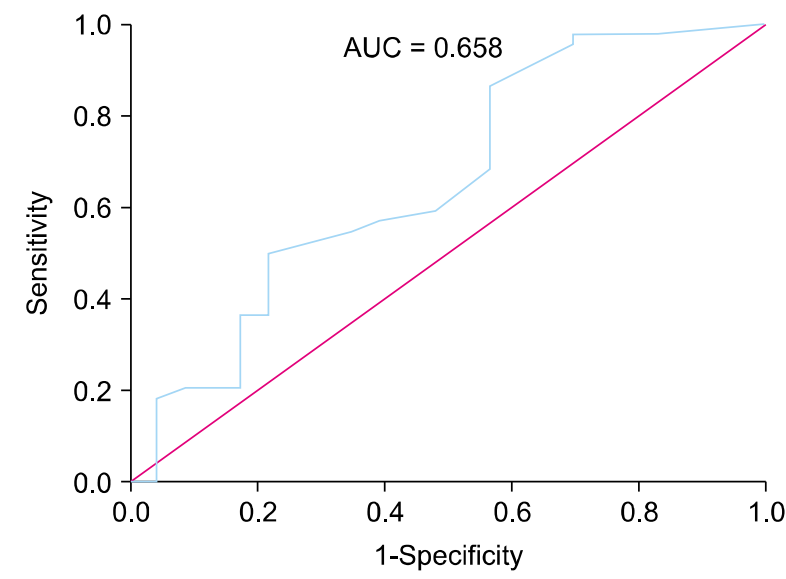

Fig. 2. Receiver operating characteristic curve. The areas under the curve (AUC) is 0.658 ( $95 \%$ confidence interval, 0.514-0.802; $P=0.035$ ). sessed by multivariate logistic regression analysis to determine the degree of the variables' association with PHN, described using the OR and $95 \%$ CI. Although old age $(\geq 65$ years) and female sex were not significantly associated in the univariate analysis, they were included in the multivariate analysis because they are well-known risk factors for development of PHN (Table 2) [8]. An SNRB performed at more than 14 days was the only significant predictor for persistent PHN for a long-term period (adjusted OR, 3.89; 95\% CI, 1.02-14.93; $P=0.047$.

Kaplan-Meier survival curves for the two groups show that the time to complete cure of PHN from an SNRB was

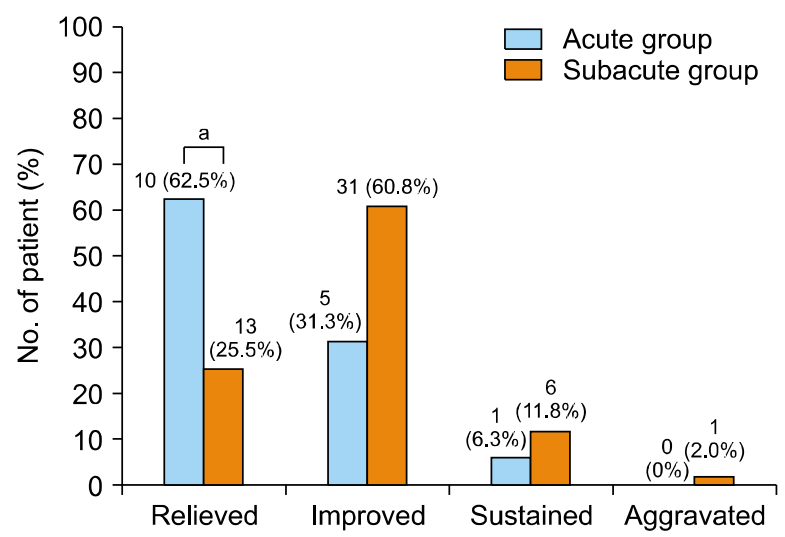

Fig. 3. Patient-reported long-term severity of postherpetic neuralgia $(\mathrm{PHN})$. Acute group: selective nerve root block $(\mathrm{SNRB}) \leq 14$ days, subacute group; SNRB $>14$ days from herpes zoster onset. Details of patients-reported 4-step severity grading of PHN: "relieved", no pain; "improved", pain is reduced but remained; "sustained", painful and no change in pain after treatment; "aggravated", more painful after treatment. ${ }^{a}$ The proportions of cured (relieved) patients were $62.5 \%$ and $25.5 \%$ in acute and subacute groups, respectively, which was significantly different by Mann-Whitney rank-sum test $(P=0.007)$.

Table 2. Logistic Regression Analysis

\begin{tabular}{|c|c|c|c|c|c|c|c|c|}
\hline \multirow{3}{*}{ Factor } & \multicolumn{4}{|c|}{ Univariate analysis } & \multicolumn{4}{|c|}{ Multivariate analysis } \\
\hline & \multirow{2}{*}{$\begin{array}{c}\text { Crude } \\
\text { OR }\end{array}$} & \multicolumn{2}{|c|}{$95 \% \mathrm{Cl}$} & \multirow{2}{*}{$P$ value } & \multirow{2}{*}{$\begin{array}{c}\text { Adjusted } \\
\text { OR }\end{array}$} & \multicolumn{2}{|c|}{$95 \% \mathrm{Cl}$} & \multirow{2}{*}{$P$ value } \\
\hline & & Low & High & & & Low & High & \\
\hline Age $\geq 65 \mathrm{yr}$ & 2.11 & 0.75 & 5.9 & 0.155 & & & & \\
\hline Female sex & 0.4 & 0.14 & 1.16 & 0.092 & & & & \\
\hline SNRB $>14$ days & 4.87 & 1.48 & 16.05 & $0.009^{*}$ & 3.89 & 1.02 & 14.93 & $0.047^{\star}$ \\
\hline
\end{tabular}

The adjusted odds ratio (OR) is adjusted for age and sex. $\mathrm{Cl}$ : confidence interval, SNRB: selective nerve root block. ${ }^{*} P<0.05$ was considered statistically significant. 


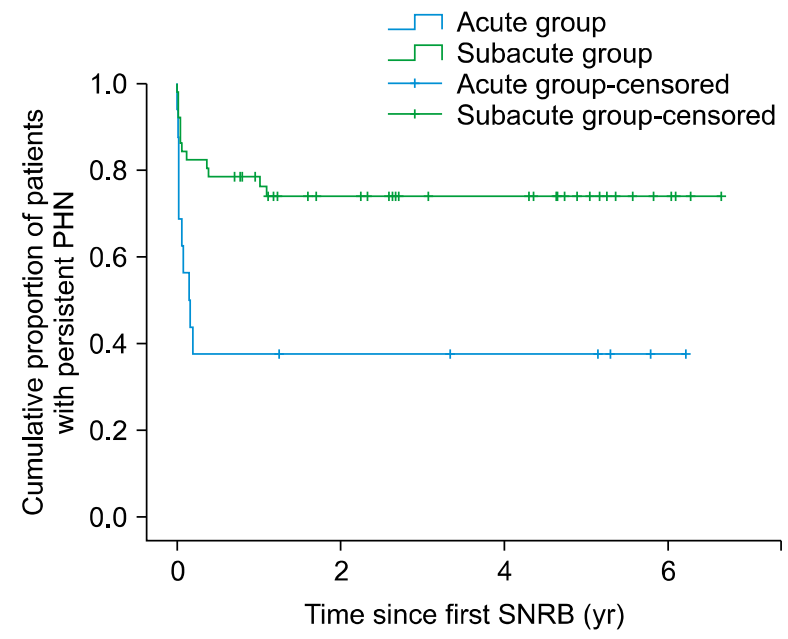

Fig. 4. Kaplan-Meier curve. Time from selective nerve root block (SNRB) to complete cure of postherpetic neuralgia $(\mathrm{PHN})$ was significantly shorter in the acute group than in the subacute group $(2.4 \pm 0.7$ years vs. $5.0 \pm 0.4$ years; $P=0.003$ by log-rank test). Acute group: SNRB $\leq 14$ days; subacute group: SNRB $>14$ days from herpes zoster onset.

significantly shorter in the acute group than in the subacute group $(2.4 \pm 0.7$ years VS. $5.0 \pm 0.4 \mathrm{yr} ; P=0.003$ by log-rank test) (Fig. 4).

\section{DISCUSSION}

This study demonstrated that the application of an early SNRB during the acute stage of $\mathrm{HZ}$ (within 14 days) decreased the incidence of PHN, and shortened the duration of PHN, with a median of 5.0 years of surveillance for HZ. The efficacy of interventional treatment, including sympathetic block and neuraxial block for preventing PHN, had been controversial. However, from recent systemic reviews and meta-analysis, evidences for several interventions, such as paravertebral blocks and continuous/repeated epidural blocks for prevention of PHN, appear to be strong $[4,9]$. In the current study, the SNRB also appears to be an effective method for improving PHN-related outcomes. The SNRB may be the theoretically most effective method of drug delivery to the DRG, which is the primary target for intervention to manage ZRP, with a small volume of injectate. Even though the pathophysiology of PHN has not been yet clearly understood, the DRG is the origin and core of the progression of PHN. The DRG contains many sensory channels and is associated with nociception and pain transmission. Reactivated HZ virus which had resided in the DRG can spread along the nerve ending and even the dorsal horn of spinal cord. And various peripheral and central sensitizations, such as spontaneous ectopic discharge, neuroinflammation and degeneration of the spinal cord dorsal horn may contribute to the progression of PHN $[10,11]$.

The incidence of PHN is reported to be about $12.5 \%-$ $38.4 \%$ in the literature, and increases with age [1,2]. And the number of patients and medical care cost associated with PHN is steadily increasing every year [3]. The increase in the aged population and extended life expectancy may contribute to this phenomenon. As our study demonstrates, PHN may persist for months or even years. PHN appeared to persist for 5.0 years according to KaplanMeier survival curves, even though the SNRB had been applied between 14 to 90 days from HZ onset. Surprisingly, an early SNRB performed within 14 days, which may mean the active stage of $\mathrm{HZ}$, dramatically shortened the duration of PHN to 2.4 years. In the current study, the authors intended to evaluate the longer term outcome of PHN. To the best of our knowledge, this is the first investigation that evaluates the efficacy of interventional treatment on the long-term progression of PHN up to 5 years.

Meanwhile, the timing of interventional treatment is another important concern to pain physicians. The consensus on timing of interventional treatment for $\mathrm{HZ}$ and PHN has not been well established, but several guidelines recommend interventional treatment as 2nd line treatment, which can be chosen only when conservative treatment fails $[11,12]$. However, early interventional treatment may be necessary, because current analgesic treatment with antidepressants, anticonvulsants, opioids, and topical agents often fails to relieve symptoms. Kim et al. [13] reported that the early transforaminal epidural injection (TFEI) during the acute stage of $\mathrm{HZ}$ (within 30 days) significantly shortens the time to ZRP relief and reduces the incidence of PHN in comparison to TFEI given during subacute stage (30 to 90 days). Although the period of ZRP can be divided into three phases, namely $\mathrm{HZ}$ acute pain (within 30 days after rash onset), subacute herpetic neuralgia (30 to 120 days) and PHN (persisting 120 days or more after rash onset), the natural course of $\mathrm{HZ}$ is diverse among patients [14]. In the current study, we compared participants in acute SNRB (within 14 days) and subacute (14 to 90 days) SNRB groups, because the cut-off value of the time 
elapsed from $\mathrm{HZ}$ onset to the first SNRB predictive of the persistent (non-cured) PHN was 14.5 days, calculated by the ROC curve assessment.

However, the authors think that the application of early intervention for the general population may be not necessary. As discussed in several studies, there are significant predictors of PHN, such as old age, severe zoster pain, severe rash, and ophthalmic involvement $[1,3,8]$. The early intervention might impact more positively for a cohort with certain risk factors than for others in the development of PHN. Larger studies with greater power to detect associations, and studies considering the socioeconomic burden associated with early intervention for PHN may be needed.

There are a few study limitations. First, the recurrence incidence of $\mathrm{HZ}$ is 12.0 per 1,000 person years with 4.4 year of the mean follow-up period, and ZRP lasting longer than 90 days, which may indicate the presence of PHN, is significantly associated with the recurrence of zoster [15]. However, in the present study, we didn't evaluate the recurrence of $\mathrm{HZ}$ on either the original or a new dermatome area. Second, drugs including local anesthetics and dexamethasone might spread along the epidural space or adjacent nerve root, even though the pain physician had intended to perform an SNRB with a small volume of injectate. Only $1 \mathrm{~mL}$ of contrast dye can spread onto adjacent nerve root in approximately $50 \%$ of patients during a lumbar SNRB [16]. However, drug spreading into a single nerve root was confirmed by using contrast dye prior to the drug injection. Third, our study is limited due to the retrospective study design and relatively small sample size.

In conclusion, that application of early SNRB during the acute stage of HZ (within 14 days) appears to decrease the incidence and shorten the duration of PHN, with a median of 5.0 years of follow-up after the initial diagnosis of $\mathrm{HZ}$.

\section{ACKNOWLEDGMENTS}

The authors would like to specially thank prof. Ju-Hyong Lee for statistical support in this study.

\section{CONFLICT OF INTEREST}

No potential conflict of interest relevant to this article was reported.

\section{ORCID}

A Ram Doo, https://orcid.org/0000-0003-1310-790X Jin-Wook Choi, https://orcid.org/0000-0001-7750-1987 Ju-Hyung Lee, https://orcid.org/0000-0003-2487-4098 Ye Sull Kim, https://orcid.org/0000-0001-8771-488X Min-Jong Ki, https://orcid.org/0000-0001-9959-7908 Young Jin Han, https://orcid.org/0000-0002-9111-0760 Ji-Seon Son, https://orcid.org/0000-0002-6672-4576

\section{REFERENCES}

1. Forbes HJ, Thomas SL, Smeeth L, Clayton T, Farmer R, Bhaskaran $\mathrm{K}$, et al. A systematic review and meta-analysis of risk factors for postherpetic neuralgia. Pain 2016; 157 : 30-54.

2. Song $H$, Lee J, Lee $M$, Choi WS, Choi JH, Lee MS, et al. Burden of illness, quality of life, and healthcare utilization among patients with herpes zoster in South Korea: a prospective clinical-epidemiological study. Int J Infect Dis 2014; 20: 23-30.

3. Hong MJ, Kim YD, Cheong YK, Park SJ, Choi SW, Hong HJ. Epidemiology of postherpetic neuralgia in Korea: an electronic population health insurance system based study. Medicine (Baltimore) 2016; 95: e3304.

4. Kim HJ, Ahn HS, Lee JY, Choi SS, Cheong YS, Kwon K, et al. Effects of applying nerve blocks to prevent postherpetic neuralgia in patients with acute herpes zoster: a systematic review and meta-analysis. Korean J Pain 2017; 30: 3-17.

5. Makharita MY, Amr YM, El-Bayoumy Y. Effect of early stellate ganglion blockade for facial pain from acute herpes zoster and incidence of postherpetic neuralgia. Pain Physician 2012; 15: 467-74.

6. Ji G, Niu J, Shi Y, Hou L, Lu Y, Xiong L. The effectiveness of repetitive paravertebral injections with local anesthetics and steroids for the prevention of postherpetic neuralgia in patients with acute herpes zoster. Anesth Analg 2009; 109: 1651-5.

7. Eckel TS, Bartynski WS. Epidural steroid injections and selective nerve root blocks. Tech Vasc Interv Radiol 2009; 12: $11-21$.

8. Forbes HJ, Bhaskaran K, Thomas SL, Smeeth L, Clayton T, Mansfield $K$, et al. Quantification of risk factors for postherpetic neuralgia in herpes zoster patients: a cohort study. Neurology 2016; 87: 94-102.

9. Kumar V, Krone K, Mathieu A. Neuraxial and sympathetic blocks in herpes zoster and postherpetic neuralgia: an appraisal of current evidence. Reg Anesth Pain Med 2004; 29: 454-61.

10. Reichelt M, Zerboni L, Arvin AM. Mechanisms of varicellazoster virus neuropathogenesis in human dorsal root ganglia. 
J Virol 2008; 82: 3971-83.

11. Jeon $\mathrm{YH}$. Herpes zoster and postherpetic neuralgia: practical consideration for prevention and treatment. Korean $J$ Pain 2015; 28: 177-84.

12. Van Zundert J, Hartrick C, Patjin J, Huygen F, Mekhail N, van Kleef M. Evidence-based interventional pain medicine according to clinical diagnoses. Pain Pract 2011; 11: 4239.

13. Kim ED, Bak HH, Jo DH, Park HJ. Clinical efficacy of transforaminal epidural injection for management of zosterassociated pain: a retrospective analysis. Skeletal Radiol 2018; 47: 253-60.
14. Dworkin RH, Gnann JW Jr, Oaklander AL, Raja SN, Schmader KE, Whitley RJ. Diagnosis and assessment of pain associated with herpes zoster and postherpetic neuralgia. J Pain 2008; 5(1 Suppl): S37-44.

15. Kim YJ, Lee CN, Lee MS, Lee JH, Lee JY, Han K, et al. Recurrence rate of herpes zoster and its risk factors: a population-based cohort study. J Korean Med Sci 2018; 34: e1.

16. Vassiliev D. Spread of contrast during $L 4$ and $L 5$ nerve root infiltration under fluoroscopic guidance. Pain Physician 2007; 10: 461-6. 